\title{
A Simple One-Dimensional Model of Constrained Recovery in Shape Memory Alloys
}

\author{
T. VIDENIC, M. BROJAN, J. KUNAVAR, and F. KOSEL \\ Department of Mechanics, Faculty of Mechanical Engineering, University of Ljubljana, Ljubljana, Slovenia
}

Received 5 January 2011; accepted 8 September 2011.

In elements made of shape memory alloys (SMAs), large stresses are generated if during heating, shape recovery associated with martensitic transformation, is constrained by an external element. This kind of recovery process is called constrained recovery. In this article, a simple one-dimensional model for the analysis of constrained recovery in SMA wire is presented. The model is based on the theory of generalized plasticity, which was developed by Lubliner and Auricchio. Despite the fact that the model considers an assumption of a non-constant Young's modulus of SMA wire, it remains simple and is well suited for further practical engineering applications and calculations. The regularity of the model is verified by comparing it to experimental results published by Kato, Inagaki, and Sasaki. It is shown that the assumption of non-constant Young's modulus significantly improves the agreement between theory and experiments.

Keywords: shape memory alloys, NiTi wire, constrained recovery, generalized plasticity

\section{Introduction}

Shape memory effect is a unique property of some alloys when, after being deformed at a lower temperature, they recover their original shape when heated to a higher temperature. The socalled "memory" is a result of reversible martensitic transformation, which is a solid-solid, diffusionless transition between a crystallographically less ordered low temperature product phase (martensite) and a crystallographically more ordered high temperature parent phase (austenite). The return to the original shape at zero stress state starts at the austenite start temperature $A_{S}$ and completes at the austenite finish temperature $A_{F}$. Vice versa, if the SMA is cooled from the austenitic phase at zero stress state, it starts to transform back to martensite at a temperature called martensite start temperature $M_{S}$ and ends at martensite finish temperature $M_{F}$.

In this way, large strains of even $10 \%$ can be recovered during heating from martensite to austenite and the process is often referred to as free recovery. However, if free recovery is hampered by an external obstacle before temperature $A_{F}$ is reached, the process is called constrained recovery and large recovery stresses up to $800 \mathrm{MPa}$ can be generated. Recovery stresses can diminish if the shape memory alloy (SMA) is then

Address correspondence to T. Videnic, Department of Mechanics, Faculty of Mechanical Engineering, University of Ljubljana, Askerceva 6, Ljubljana 1000, Slovenia. E-mail: tomaz.videnic@ fs.uni-lj.si

Color versions of one or more of the figures in the article can be found online at www.tandfonline.com/umcm. cooled and transformation from austenite to martensite occurs. This property makes SMAs ideally suited for use as fasteners, seals, connectors, and clamps, not only in industrial applications [1-3], but also in medicine [3-5]. The same property is used when SMA elements are embedded into a composite matrix for shape control of structural elements [6, 7]. Of particular importance for such applications is an understanding of the generation of recovery stresses with respect to temperature. Available publications on the process of constrained recovery in SMAs are mostly limited to uni-axial examples [8-17], with rare exceptions where constrained recovery in SMA rings is dealt with $[18,19]$. Because of the complexity of the problem, some simplifications are often used in mathematical modeling. One of them is considering the value of Young's modulus of SMA for martensite and austenite phase as constant, though it is well known that this value differs significantly.

The principal aim of the present article is to develop a simple mathematical model of uni-axial constrained recovery in an SMA wire element considering different values of Young's modulus for the martensite and austenite phase. The model is based on the theory of generalized plasticity which, despite its simplicity, predicts the shape-memory effect and superelastic behavior. It was developed by Lubliner and Auricchio [20, 21]. The external obstacle that causes constrained recovery in the SMA wire is an elastically deformable bias spring made of conventional material. The data for the NiTi SMA wire containing 50.0 at.\% titanium and for the steel bias spring were fed into the mathemetical model in order to generate a thermomechanical response. The results of the modeling were compared with experimental tests published by Kato et al. [15]. The comparison of the theory and experiments show 
that assuming a non-constant Young's modulus of martensite and austenite essentially improves the theory.

This article outlines first some general aspects of the process of constrained recovery in SMAs, then the model is presented in detail, and finally several numerical examples and a comparison between theory and experiments are examined.

\section{Process of Uni-Axial Constrained Recovery}

Large stresses are generated in an element made of SMA, if the recovery to austenite structure during heating is constrained by an external element. In this article, the shape memory element is represented by an SMA wire and the external element by a linear bias spring made of conventional material.

The SMA wire is cooled from the austenite state, $T>A_{F}$, to the martensite state, $T=T_{0}<M_{F}$. It is assumed that the total strain of the SMA wire is zero at temperature $T_{0}, \varepsilon_{S}=0$, and the length is $L_{0}$. The SMA wire is then loaded by a tensile force and unloaded at constant temperature $T=T_{0}<M_{F}$ so that the total strain after unloading is $\varepsilon_{S}=\varepsilon_{S 0}$ and the length is $L_{S 0}$. The recoverable shape memory strain $\varepsilon_{S 0}$ will be called prestrain and can disappear completely when the SMA wire is heated above temperature $A_{F}$. The process is called free recovery. If recovery is hampered by an external obstacle, the process is called constrained recovery and prestrain $\varepsilon_{S 0}$ disappears only when temperature $A_{F}^{\sigma}>A_{F}$ is reached. The system SMA wire-external obstacle can then be further heated or cooled. If it is cooled to the temperature $T<M_{S}^{\sigma}$, transformation from austenite to martensite can occur again and recovery stresses diminish. The temperatures $A_{F}^{\sigma}$ and $M_{S}^{\sigma}$ can be called, respectively, austenite finish and martensite start temperature under stress $\sigma$. The process of constrained recovery is schematically shown in Figure 1, where a linear bias spring made of a conventional material (e.g., steel) presents an external obstacle.

In Figure $1, L_{k 0}$ is spring length at temperature $T_{0}, L_{k}$ is length of the spring at temperature $T, L_{S}$ is length of the SMA wire at temperature $T$, and $T_{C}$ is contact temperature at which the SMA wire and the spring make contact.

The complete process of constrained recovery can be divided into five temperature ranges, as proposed by Stalmans et al. [12]: (a) After the loading-unloading cycle and during (a)

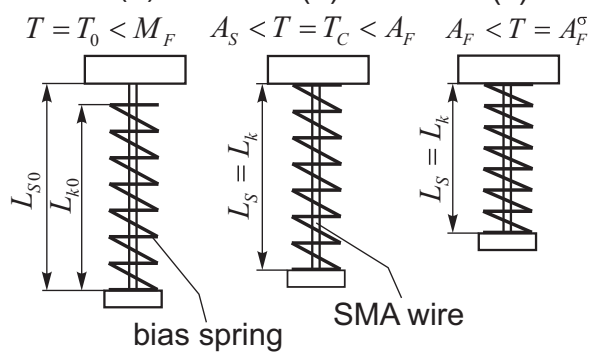

(d)

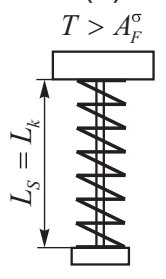

Fig. 1. Process of uni-axial constrained recovery. heating the SMA wire has martensite structure between the temperatures $T_{0}$ and $A_{S}$. Both elements (the SMA wire and the bias spring) are extending due to heating. (b) At temperature $A_{S}$, the retransformation in the SMA wire starts so that the wire contracts during heating while the bias spring extends. At temperature $T_{C}$ both elements touch each other and the process of constrained recovery begins. (c) Above temperature $T_{C}$, retransformation to austenite in the SMA wire is constrained because of the bias spring and continues until temperature $A_{F}^{\sigma}$ is reached. Since stresses in the SMA wire increase, the temperature $A_{F}^{\sigma}$ at which the retransformation to austenite is completed is higher than $A_{F}$. (d) If the system is heated above temperature $A_{F}^{\sigma}$, the SMA wire is in the austenite region and behaves as conventional material; like a bias spring it extends with increasing temperature. If the system is cooled, there are two possibilities. If temperature is above $M_{S}^{\sigma}$, then SMA wire behaves as conventional material. (e) But if during cooling, the temperature is below $M_{S}^{\sigma}$, then transformation from austenite to martensite takes place, the SMA wire extends, and recovery stresses diminish. At some temperature $T_{\text {loose }}$, there is no contact between both elements anymore and recovery stresses drop to zero.

The expression for the total strain of the SMA wire $\varepsilon_{S}$ can be written in two different ways:

$$
\begin{aligned}
& \varepsilon_{S}=\frac{L_{S}-L_{0}}{L_{0}}, \\
& \varepsilon_{S}=\varepsilon_{S R}+\varepsilon_{T H}+\varepsilon_{E L}=\varepsilon_{S R}+\alpha_{S}\left(T-T_{0}\right)+\frac{\sigma}{E},
\end{aligned}
$$

where $\varepsilon_{S R}$ is the recoverable shape memory strain, $\varepsilon_{T H}$ is thermal strain, $\varepsilon_{E L}$ is elastic strain, $\alpha_{S}$ is linear thermal dilatation coefficient, $\sigma$ is stress, and $E$ is Young's modulus of the SMA wire. In the literature [20], the recoverable strain $\varepsilon_{S R}$ is usually taken as proportional to the mass fraction of martensite $\xi$ :

$$
\varepsilon_{S R}=\varepsilon_{S 0} \xi
$$

with $\xi=0$ denoting all austenite and $\xi=1$ all martensite structure of SMA wire. After the loading-unloading cycle at temperature $T_{0}$, the length $L_{S 0}$ can be calculated from Eqs. (1) and (3): $L_{S 0}=L_{0}\left(1+\varepsilon_{S 0}\right)$.

Experiments clearly indicate that the Young's modulus $E$ of the SMA materials is strongly dependent on the mass fraction of martensite $\xi$. An acceptable assumption for the modulus function of an SMA material is, as proposed in the literature [11]:

$$
E=\left(E_{M}-E_{A}\right) \xi+E_{A}
$$

where $E_{M}$ is the modulus value for the SMA as $100 \%$ martensite and $E_{A}$ is the modulus value for the SMA as $100 \%$ austenite. The ratio of the magnitudes of $E_{A}$ to $E_{M}$ usually has a value of 2 or even greater.

The expression for the total strain of the spring $\varepsilon_{k}$ can be written in a similar way as Eq. (1):

$$
\varepsilon_{k}=\frac{L_{k}-L_{k 0}}{L_{k 0}} .
$$




\section{The Mathematical Model of Constrained Recovery}

It is assumed that the SMA wire is first cooled from the austenite to martensite state then loaded by tensile force and unloaded at constant temperature $T_{0}$. The result of the loadingunloading cycle is the prestrain $\varepsilon_{S 0}$, which can be recovered by heating. Five temperature regions can be distinguished during heating, starting from $T_{0}$.

\subsection{First Temperature Range, $T_{0} \leq T \leq A_{S}$}

Both elements are extending during heating and the recovery in the SMA wire from martensite to austenite has not started yet. The total strain of the SMA wire $\varepsilon_{S}$ can be written from Eq. (2) in the following way:

$$
\varepsilon_{S}=\varepsilon_{S 0}+\alpha_{S}\left(T-T_{0}\right) .
$$

In a similar way, the total strain of the bias spring $\varepsilon_{k}$ can be written as:

$$
\varepsilon_{k}=\alpha\left(T-T_{0}\right),
$$

where $\alpha$ is linear thermal expansion coefficient of the bias spring. The stress state in both elements is zero, since the SMA wire and bias spring do not touch each other in this temperature range.

\subsection{Second Temperature Range, $A_{S} \leq T \leq T_{C}$}

In the SMA wire, a reverse transformation from martensite to austenite begins at temperature $A_{S}$. The total strain of the SMA wire can be written from Eq. (2),

$$
\varepsilon_{S}=\varepsilon_{S R}+\alpha_{S}\left(T-T_{0}\right) .
$$

Equation (8) is very similar to Eq. (6) except that the recoverable shape memory strain $\varepsilon_{S R}$ is no longer constant but diminishes with increasing temperature $T$. Another equation (the flow rule), which would relate martensite mass fraction $\xi$ (or recoverable shape memory strain $\varepsilon_{S R}$ ), to temperature $T$ and stress $\sigma$ in the SMA wire is needed. It can be deduced from the theory of generalized plasticity, which was developed by Lubliner and Auricchio [20,21]. Different flow rules can be assumed. In our case, the linear flow rule for retransformation from martensite to austenite will be used:

$$
\sigma=C\left[T-A_{F}+\xi\left(A_{F}-A_{S}\right)\right],
$$

where $C$ is one of the fundamental descriptors of SMA and is called a stress rate. Since the stress in the SMA wire is still zero, $\sigma=0$, and using Eq. (3) it can be written from Eq. (9):

$$
\varepsilon_{S R}=\varepsilon_{S 0} \frac{A_{F}-T}{A_{F}-A_{S}} .
$$

Recoverable shape memory contact strain of the SMA wire $\varepsilon_{C}$ at contact temperature $T_{C}$ can be calculated from the condition that the lengths of both elements are equal, $L_{S}\left(T_{C}\right)=L_{k}\left(T_{C}\right)=L_{C}$ :

$$
\begin{aligned}
L_{S}\left(T_{C}\right) & =L_{0}\left[1+\varepsilon_{C}+\alpha_{S}\left(T_{C}-T_{0}\right)\right] \\
& =L_{k 0}\left[1+\alpha\left(T_{C}-T_{0}\right)\right]=L_{k}\left(T_{C}\right)=L_{C} .
\end{aligned}
$$

Contact mass fraction of martensite $\xi_{C}$ can be easily calculated from Eq. (3): $\xi_{C}=\varepsilon_{C} / \varepsilon_{S 0}$. The contact temperature $T_{C}$ can be calculated from Eq. (10):

$$
T_{C}=A_{F}-\frac{\varepsilon_{C}}{\varepsilon_{S 0}}\left(A_{F}-A_{S}\right) .
$$

If $\varepsilon_{C}=0\left(\xi_{C}=0\right)$, then contact temperature is $T_{C}=A_{F}$ and there is no constrained recovery. On the other hand, if $\varepsilon_{C}=\varepsilon_{S 0}$ $\left(\xi_{C}=1\right)$, the contact temperature is $T_{C}=A_{S}$, which means that the process of constrained recovery begins at the same time as the retransformation from martensite to austenite and stresses in both elements are maximal.

\subsection{Third Temperature Range, $T_{C} \leq T \leq A_{F}^{\mathrm{g}}$}

From temperature $T_{C}$ onward the SMA wire and the bias spring are in contact and in both elements stresses are increasing with increasing temperature $T$ (recoverable shape memory strain $\varepsilon_{S R}$ is decreasing and the bias spring resists contraction). The SMA wire-bias spring system shown in Figure 2 has to be in static equilibrium: $F_{S}=F_{k}$.

The force in the bias spring $F_{k}$ can be written:

$$
F_{k}=k\left[L_{C}\left(1+\alpha\left(T-T_{C}\right)\right)-L_{k}\right],
$$

where $k$ is the bias spring constant. By considering the equilibrium equation, Eqs. (1) and (13), and equal lengths of the SMA wire and the bias spring, the total strain of the SMA wire $\varepsilon_{S}$ can be written in the following form:

$$
\varepsilon_{S}=C_{1}+C_{2} T-C_{3} \sigma,
$$

where $C_{1}, C_{2}$, and $C_{3}$ are the constants:

$$
\begin{aligned}
& C_{1}=\left(L_{C}\left(1-\alpha T_{C}\right)-L_{0}\right) / L_{0}, \\
& C_{2}=\alpha T_{C} / L_{0}, \\
& C_{3}=Q /\left(k L_{0}\right),
\end{aligned}
$$

where $Q$ is the SMA wire's cross section. The total strain of the SMA wire $\varepsilon_{S}$ can also be written in another form from

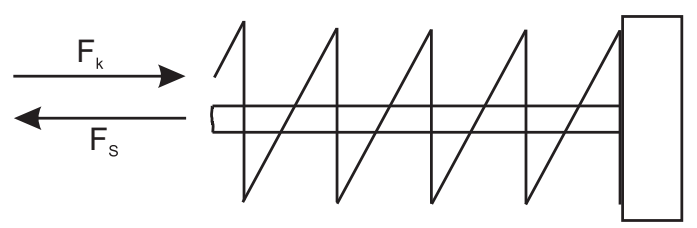

Fig. 2. Forces in the SMA wire and bias spring. 
Eq. (2):

$$
\varepsilon_{S}=\varepsilon_{S 0} \xi+\alpha_{S}\left(T-T_{0}\right)+\frac{\sigma}{\left(E_{M}-E_{A}\right) \xi+E_{A}},
$$

where Eqs. (3) and (4) were used. In Eqs. (14) and (16), the flow rule (9) can be used and then both expressions can be equalized. After some rearrangements, a simple quadratic function can be written:

$$
a_{1} \xi^{2}-a_{2}(T) \xi-a_{3}(T)=0
$$

where $a_{1}, a_{2}(T)$, and $a_{3}(T)$ are:

$$
\begin{aligned}
a_{1}= & \left(E_{A}-E_{M}\right)\left[\varepsilon_{S 0}+C_{3} C\left(A_{F}-A_{S}\right)\right] \\
a_{2}(T)= & E_{A}\left[\varepsilon_{S 0}+C_{3} C\left(A_{F}-A_{S}\right)\right]+\left(E_{A}-E_{M}\right) \\
& \times\left[C_{1}+C_{2} T-C_{3} C\left(T-A_{F}\right)-\alpha_{S}\left(T-T_{0}\right)\right] \\
& +C\left(A_{F}-A_{S}\right), \\
a_{3}(T)= & E_{A}\left[\alpha_{S}\left(T-T_{0}\right)-C_{1}-C_{2} T+C_{3} C\left(T-A_{F}\right)\right] \\
& +C\left(T-A_{F}\right) .
\end{aligned}
$$

The solution for Eq. (17) yields two distinct real roots for $\xi$. However, since $\xi_{C} \geq \xi \geq 0$, the only acceptable root is given by:

$$
\xi(T)=\frac{a_{2}(T)-\sqrt{a_{2}^{2}(T)+4 a_{1} a_{3}(T)}}{2 a_{1}} .
$$

When the mass fraction of martensite $\xi$ is known, stress $\sigma$ in the SMA wire can be easily calculated from Eq. (9). The temperature $A_{F}^{\sigma}$ at which retransformation from martensite to austenite during constrained recovery is completed can be calculated from Eq. (17) with the condition $\xi\left(A_{F}^{\sigma}\right)=0 \Rightarrow$ : $a_{3}\left(A_{F}^{\sigma}\right)=0$

$$
A_{F}^{\sigma}=\frac{C A_{F}+E_{A}\left(\alpha_{S} T_{0}+C_{1}+C_{3} C A_{F}\right)}{C+E_{A}\left(\alpha_{S}-C_{2}+C_{3} C\right)} .
$$

Stress at the temperature $A_{F}^{\sigma}$ can then be calculated from Eq. (9):

$$
\sigma\left(A_{F}^{\sigma}\right)=C E_{A} \frac{C_{1}+C_{2} A_{F}-\alpha_{S}\left(A_{F}-T_{0}\right)}{C+E_{A}\left(\alpha_{S}-C_{2}+C_{3} C\right)} .
$$

The length of the SMA wire and the length of the bias spring are equal at temperature $A_{F}^{\sigma}$ and can be calculated from Eq. (14):

$$
L_{S}\left(A_{F}^{\sigma}\right)=L_{0}\left(1+C_{1}+C_{2} A_{F}^{\sigma}-C_{3} \sigma\left(A_{F}^{\sigma}\right)\right)=L_{k}\left(A_{F}^{\sigma}\right) .
$$

If the bias spring is regarded as an ideally rigid or nondeformable body, it can be achieved in the model by using constants $\alpha=0$ and $k=\infty\left(C_{2}=0, C_{3}=0\right)$. In this case, the length of both elements is constant: $L\left(A_{F}^{\sigma}\right)=$ $L_{0}\left[1+\varepsilon_{C}+\alpha_{S}\left(T_{C}-T_{0}\right)\right]=L_{k 0}$.

\subsection{Fourth Temperature Range, $A_{F}^{\sigma} \leq T$ or $A_{F}^{\sigma} \geq T \geq M_{S}^{\sigma}$}

The SMA wire and the bias spring are still in contact, but the retransformation in the SMA wire has finished and both elements extend with increasing temperature or contract while cooling. The total strain of the SMA wire $\varepsilon_{S}$ can be written from Eq. (2):

$$
\varepsilon_{S}=\alpha_{S}\left(T-T_{0}\right)+\frac{\sigma}{E_{A}} .
$$

Since Eq. (14) is still valid, it is possible to equalize Eqs. (14) and (23), and after some rearrangements, stress $\sigma$ in the SMA wire can be written as:

$$
\sigma=\frac{E_{A}}{1+C_{3} E_{A}}\left[C_{1}+\alpha_{S} T_{0}+\left(C_{2}-\alpha_{S}\right) T\right] .
$$

The length of both elements can be calculated from Eqs. (1) and (23):

$$
L_{S}=L_{k}=L_{0}\left[1+\alpha_{S}\left(T-T_{0}\right)+\frac{\sigma}{E_{A}}\right] .
$$

If the bias spring is considered as an ideally rigid body, i.e., $\alpha=$ 0 and $k=\infty$, then the stress and length of the SMA wire in the fourth temperature range are: $\sigma=E_{A}\left[C_{1}-\alpha_{S}\left(T-T_{0}\right)\right]$ and $L_{S}=L_{0}\left[1+\varepsilon_{C}+\alpha_{S}\left(T_{C}-T_{0}\right)\right]=L_{k 0}$.

\subsection{Fifth Temperature Range, $M_{S}^{v} \geq T \geq T_{\text {loose }}$}

During cooling a transformation from austenite to martensite in the SMA wire begins at temperature $M_{S}^{\sigma}$. Because of the transformation the SMA wire extends during cooling while the bias spring is contracting. Even though stresses in both elements diminish, they are still in contact so that Eqs. (14), (15), and (16) are still valid, but the flow rule (9) is not correct in this temperature range. According to the theory of generalized plasticity $[20,21]$, the linear flow rule for a transformation from austenite to martensite can be used:

$$
\sigma=C\left[T-M_{F}-(1-\xi)\left(M_{S}-M_{F}\right)\right] .
$$

In Eqs. (14) and (16), the flow rule (26) can be used and then both expressions can be equalized:

$$
\begin{aligned}
\varepsilon_{S 0} \xi & +\alpha_{S}\left(T-T_{0}\right)+\frac{C\left[T-M_{F}-(1-\xi)\left(M_{S}-M_{F}\right)\right]}{\left(E_{M}-E_{A}\right) \xi+E_{A}} \\
& =C_{1}+C_{2} T-C_{3} C\left[T-M_{F}-(1-\xi)\left(M_{S}-M_{F}\right)\right] .
\end{aligned}
$$

There are two unknowns in Eq. (27): temperature $T$ and martensite mass fraction $\xi$. Temperature $M_{S}^{\mathrm{s}}$ can be calculated from Eq. (27) using the condition $\xi=0$ :

$$
M_{S}^{\sigma}=\frac{C M_{S}+E_{A}\left(\alpha_{S} T_{0}+C_{1}+C_{3} C M_{S}\right)}{C+E_{A}\left(\alpha_{S}-C_{2}+C_{3} C\right)} .
$$


Table 1. Material Properties

\begin{tabular}{llll}
\hline$M_{S}=55^{\circ} \mathrm{C}$ & $M_{F}=17^{\circ} \mathrm{C}$ & $A_{S}=25^{\circ} \mathrm{C}$ & $A_{F}=61^{\circ} \mathrm{C}$ \\
$T_{0}=15^{\circ} \mathrm{C}$ & $L_{0}=35 \mathrm{~mm}$ & $C=5.7 \mathrm{MPaK}^{-1}$ & $\alpha_{S}=1.1 \cdot 10^{-5} \mathrm{~K}^{-1}$ \\
$E_{M}=30 \mathrm{GPa}$ & $E_{A}=70 \mathrm{GPa}$ & $E=40 \mathrm{GPa}=$ const. & $\alpha=2 \cdot 10^{-5} \mathrm{~K}^{-1}$ \\
\hline
\end{tabular}

Stress $\sigma\left(M_{S}^{\sigma}\right)$ at which a transformation from austenite to martensite begins can be calculated by substituting Eq. (28) into Eq. (26):

$$
\sigma\left(M_{S}^{\sigma}\right)=C E_{A} \frac{C_{1}+C_{2} M_{S}-\alpha_{S}\left(M_{S}-T_{0}\right)}{C+E_{A}\left(\alpha_{S}-C_{2}+C_{3} C\right)} .
$$

Equations (28) and (29) can be compared to Eqs. (20) and (21), where similar structure can be observed.

Temperature $T_{\text {loose }}$ and martensite mass fraction $\xi_{\text {loose }}$ at which stresses drop to zero and there is no contact between the SMA wire and the bias spring can also be calculated. First, the relation between $T_{\text {loose }}$ and $\xi_{\text {loose }}$ can be derived from flow rule (26) using the condition $\sigma=0$ :

$$
T_{\text {loose }}=M_{S}-\xi_{\text {loose }}\left(M_{S}-M_{F}\right)
$$

Further, it can be written from Eq. (27):

$$
\varepsilon_{S 0} \xi_{\text {loose }}+\alpha_{S}\left(T_{\text {loose }}-T_{0}\right)=C_{1}+C_{2} T_{\text {loose }} .
$$

Both unknowns can be easily calculated from Eqs. (30) and (31):

$$
\begin{aligned}
& T_{\text {loose }}=\frac{\varepsilon_{S 0} M_{S}-\left(C_{1}+\alpha_{S} T_{0}\right)\left(M_{S}-M_{F}\right)}{\varepsilon_{S 0}+\left(C_{2}-\alpha_{S}\right)\left(M_{S}-M_{F}\right)}, \\
& \xi_{\text {loose }}=\frac{C_{1}+C_{2} M_{S}-\alpha_{S}\left(M_{S}-T_{0}\right)}{\varepsilon_{S 0}+\left(C_{2}-\alpha_{S}\right)\left(M_{S}-M_{F}\right)}
\end{aligned}
$$

Equations (32) and (33) indicate that both $T_{\text {loose }}$ and $\xi_{\text {loose }}$ are independent of the Young's modulus of the SMA wire and the spring constant of the bias spring. It should also be noted that martensite mass fraction $\xi_{\text {loose }}$ is not equal to 1 in general. It means that transformation from austenite to martensite can be active also when both elements are no longer in contact, if

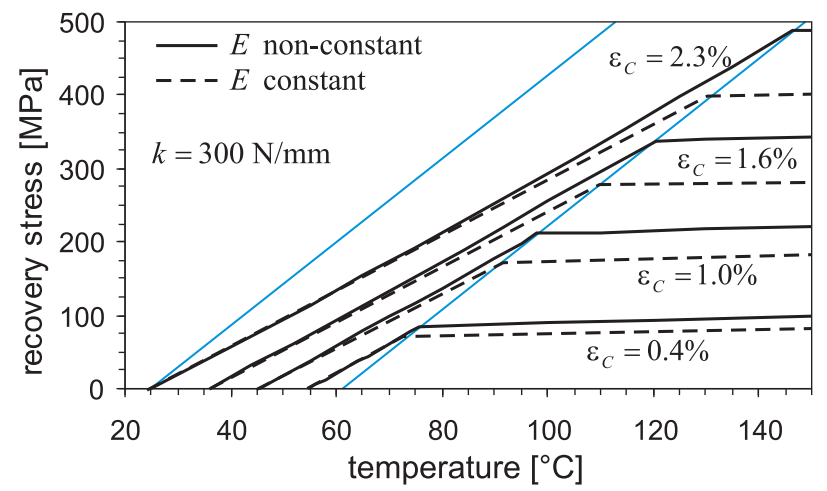

the SMA wire is further cooled to a temperature $T \leq T_{\text {loose }}$. In this case, the situation is similar as in the second temperature range where the transformation from martensite to austenite instead of the transformation from austenite to martensite is taking place. Due to the simplicity of the derivation, further cooling $T \leq T_{\text {loose }}$ will not be treated here.

When extreme values of the martensite mass fraction in this temperature range are known, $0 \leq \xi \leq \xi_{\text {loose }}$, the temperature $T$ at an appropriate value of $\xi$ can easily be calculated from Eq. (27).

\section{Numerical and Experimental Results}

Most of the material properties of the SMA wire made of NiTi alloy containing 50.0 at.\% titanium and of the external obstacle are taken from [15] and are collected in Table 1. A cold-drawn SMA wire was cut into specimens that were annealed at $400{ }^{\circ} \mathrm{C}$ for $60 \mathrm{~min}$. During the annealing, each specimen was put into a ceramic tube to remember the straight shape. The diameter of the SMA wire was $d=0.7 \mathrm{~mm}$, and the gauge length was $L_{0}=35 \mathrm{~mm}$. The average grain size was $50 \mu \mathrm{m}$ in diameter. Young's moduli for the martensite and austenite phase, $E_{M}=30 \mathrm{GPa}$ and $E_{A}=70 \mathrm{GPa}$, are taken from the literature [22]. Since the influence of non-constant and constant value of Young's modulus of the SMA wire on numerical results will also be analyzed, the constant value will be taken as $E=40 \mathrm{GPa}=$ const. The same value was used by Kato et al. in their work [15]. In all calculations, data from Table 1 were used.

The influence of different contact strains $\varepsilon_{C}$ is shown in Figure 3, while prestrain $\varepsilon_{S 0}=2.3 \%$ is constant and bias spring constant is $k=300 \mathrm{~N} / \mathrm{mm}$. The difference between constant and non-constant Young's modulus of the SMA wire

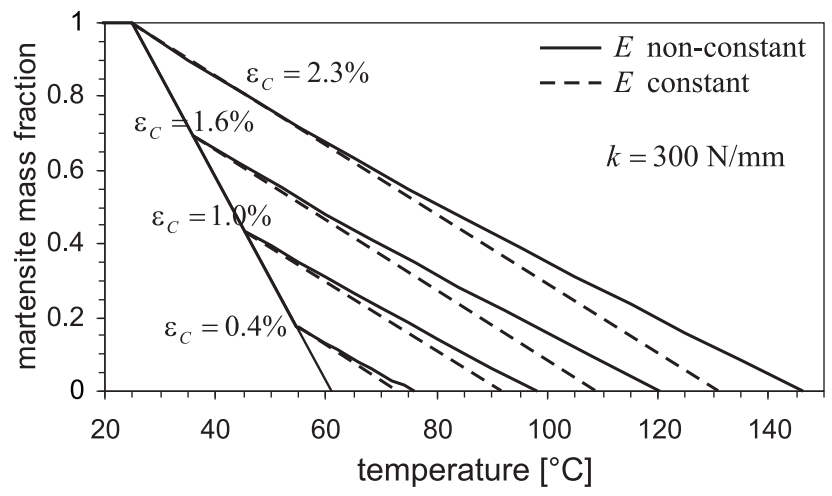

Fig. 3. The relationships between temperature $T$ and stress $\sigma$ in SMA wire and between temperature and martensite mass fraction $\xi$ at different contact strains $\varepsilon_{C}$. 


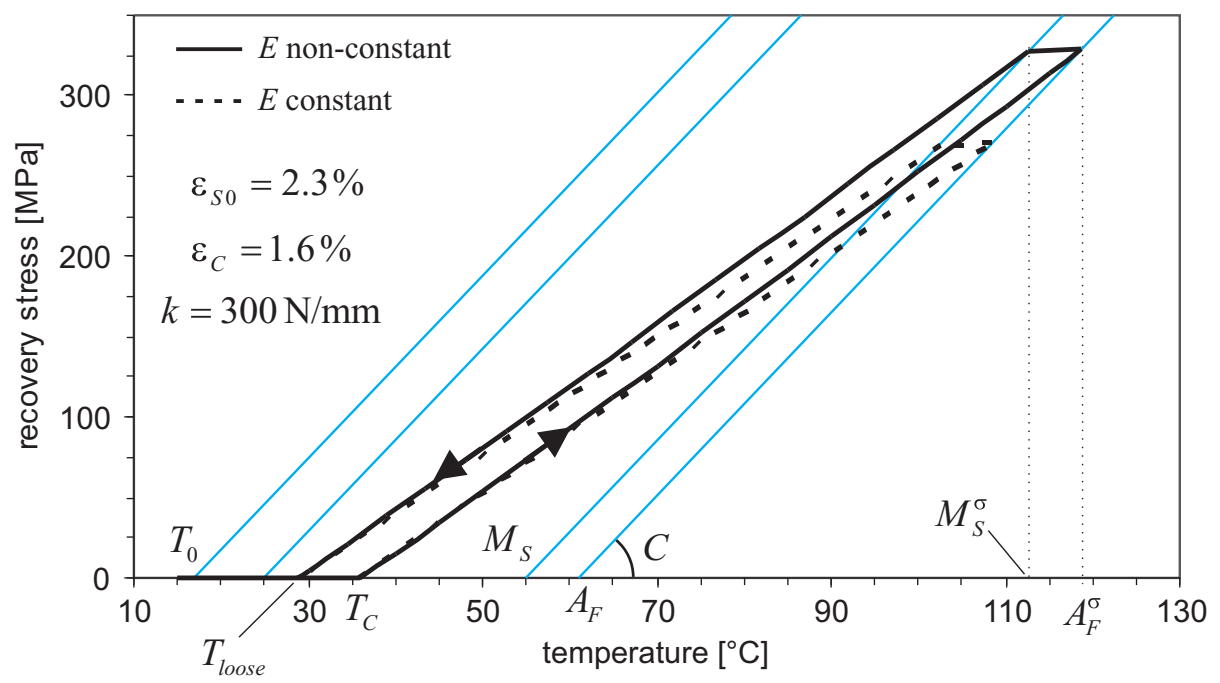

Fig. 4. Hysteresis loop of the system SMA wire-bias spring.

can be clearly seen and is increasing at higher values of contact strain $\varepsilon_{C}$.

For instance, at contact strain $\varepsilon_{C}=2.3 \%$ and if a nonconstant Young's modulus is considered, stress $\sigma\left(A_{F}^{\sigma}\right)$ and temperature $A_{F}^{\sigma}$ at which retransformation in SMA wire ends are $\sigma\left(A_{F}^{\sigma}\right)=473.7 \mathrm{MPa}$ and $A_{F}^{\sigma}=144.1^{\circ} \mathrm{C}$. But, if a constant value of Young's modulus is assumed, stress is $\sigma\left(A_{F}^{\sigma}\right)=389.1 \mathrm{MPa}$ and temperature is $A_{F}^{\sigma}=129.3^{\circ} \mathrm{C}$. In the fourth temperature range, $T \geq A_{F}^{\sigma}$, when retransformation in SMA wire is complete, stresses are still increasing $\left(\alpha>\alpha_{S}\right)$, but at much lower rates than in the third temperature range where retransformation from martensite to austenite is active.

The entire heating-cooling cycle is presented in Figure 4. Prestrain of the SMA wire is $\varepsilon_{S 0}=2.3 \%$, contact strain is $\varepsilon_{C}=1.6 \%$, and bias spring constant is $k=300 \mathrm{~N} / \mathrm{mm}$.

Recovery stresses increase rapidly during a transformation from martensite to austenite in the third temperature range, the stresses remain almost constant after transformation is complete in the fourth temperature range, and as the system is further cooled the stresses decrease rapidly in the region of austenite to martensite transformation in the fifth temperature range. A typical SMA hysteresis loop can be observed. The width of the hysteresis cycle is small since the difference between transformation temperatures $M_{S}$ and $A_{F}$ is small (see Table 1). It is interesting to note that temperature $T_{\text {loose }}$ is identical no matter if constant or non-constant Young's modulus of the SMA wire is used.

If the bias spring is considered as an ideally rigid body, then its linear thermal expansion coefficient is $\alpha=0$ and the spring constant is $k=\infty$. In this case, numerical results are collected in Figure 5.

Prestrain of the SMA wire is $\varepsilon_{S 0}=2.3 \%$ and its contact strain is taken as $\varepsilon_{C}=0.8 \%$. The difference between constant and non-constant Young's modulus of the SMA wire is even more evident. For instance, if non-constant Young's modulus is considered, stress $\sigma\left(A_{F}^{\sigma}\right)$ and temperature $A_{F}^{\sigma}$ are $\sigma\left(A_{F}^{\sigma}\right)=484.9 \mathrm{MPa}$ and $A_{F}^{\sigma}=146.1^{\circ} \mathrm{C}$. But, if constant value of Young's modulus is assumed, stress is $\sigma\left(A_{F}^{\sigma}\right)=292.0 \mathrm{MPa}$ and temperature is $A_{F}^{\sigma}=112.2^{\circ} \mathrm{C}$. In the fourth temperature range, $T \geq A_{F}^{\sigma}$, stresses are decreasing, which is physically correct since $\alpha<\alpha_{S}$.
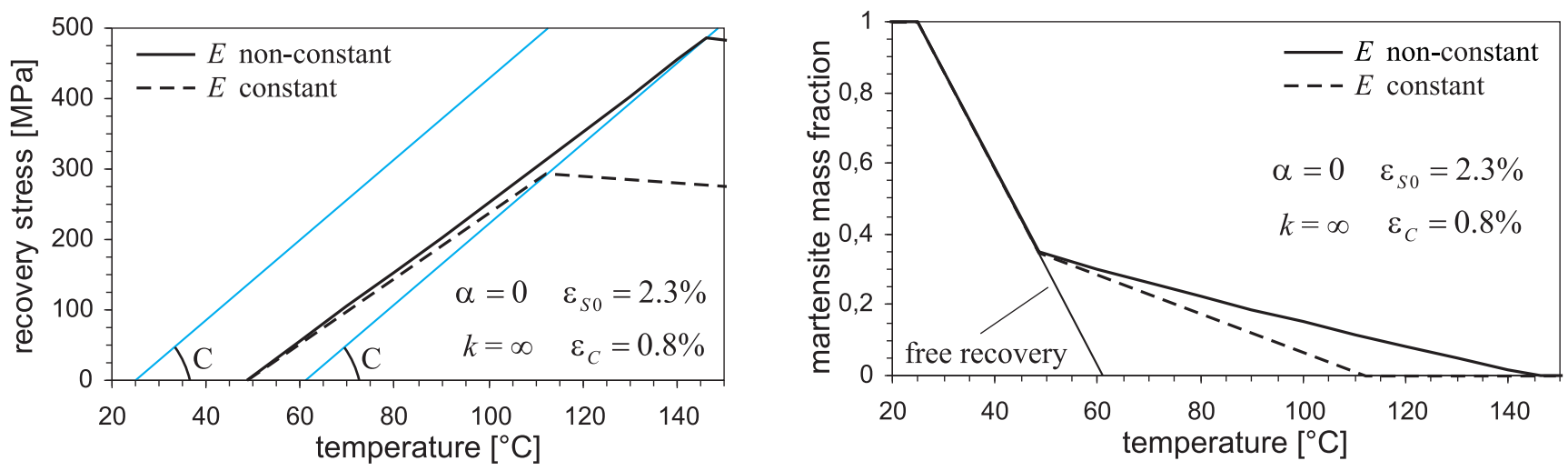

Fig. 5. The relationships between temperature $T$ and stress $\sigma$ in SMA wire and between temperature and martensite mass fraction $\xi$ when the bias spring is an ideally rigid body. 


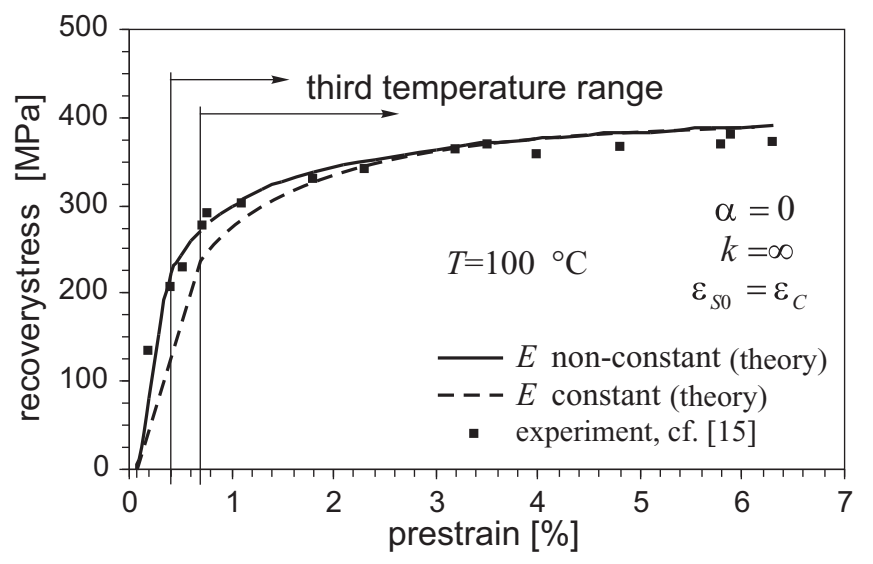

Fig. 6. Comparison of experimental and theoretical recovery stresses $\sigma$ in SMA wire at different prestrains $\varepsilon_{S 0}$ and at constant temperature $T=100^{\circ} \mathrm{C}$.

The results from the model are also compared with the experimental tests performed by Kato et al. [15] and are presented in Figure 6.

In their tests, SMA wire made of NiTi alloy containing 50.0 at.\% titanium was used. The external obstacles were crossheads of a hard-type testing machine. The SMA wire was inserted into an Instron hard-type tensile testing machine and heated at temperature $T=100^{\circ} \mathrm{C}$, which is above $A_{F}=61^{\circ} \mathrm{C}$. Since the movement of crossheads was halted during heating, the external obstacle can be regarded as an ideally rigid body. The SMA wire wanted to shrink from the length $L_{S 0}$ to $L_{0}$, but the crossheads held the constant length $L_{S 0}$ during heating and high stresses were generated in the SMA wire. Kato et al. measured the magnitudes of the shape recovery stresses $\sigma$ at different amounts of prestrain $\varepsilon_{S 0}$; contact strains $\varepsilon_{C}$ were the same as prestrains and at a constant temperature $T=100^{\circ} \mathrm{C}$. The agreement between the experiments and the theory is much better in the case of a non-constant value of Young's modulus when prestrains are $\varepsilon_{S 0}<1.5 \%$. The difference caused by the use of constant or non-constant value of Young's modulus almost disappears when prestrains are $\varepsilon_{S 0}>3 \%$. Two different domains can be seen in Figure 6. In the case of non-constant Young's modulus, the critical value of prestrain is $\varepsilon_{S 0}=0.40 \%$ and in the case of constant value the crititical value is $\varepsilon_{S 0}=0.64 \%$. When prestrain is smaller than the critical value, the retransformation to austenite in the SMA wire has completed before temperature $T=100^{\circ} \mathrm{C}$ has been reached. This means that the SMA wire is in the fourth temperature range, $\xi=0$. But if prestrain is higher than the critical value, the retransformation to austenite has not completed at temperature $T=100^{\circ} \mathrm{C}$ yet and there is a mixture of martensite and austenite, and the SMA wire is in the third temperature range, $\xi>0$. For instance, at prestrain $\varepsilon_{S 0}=3.2 \%$, the calculated value of martensite mass fraction in the case of non-constant Young's modulus is $\xi=0.70$ and in the case of constant Young's modulus is $\xi=0.69$ and stresses are also very similar. The reason for such behavior lies in the fact that at higher prestrains the effect of non-constant value of Young's modulus is reduced since the mixture in the SMA wire is $70 \%$ martensite and only $30 \%$ austenite. At lower values of prestrain, the mixture in the SMA wire is mainly austenite and the effect of non-constant Young's modulus should not be neglected.

\section{Conclusions}

A simple one-dimensional model of constrained recovery assuming a non-constant Young's modulus was developed and compared with experimental results from the literature [15]. It can be concluded that the assumption of a non-constant Young's modulus considerably improves the agreement between theory and experiments in the case when reverse transformation from martensite to austenite is completed (fourth temperature range) or when reverse transformation is not completed yet but the structure is mainly austenitic. The main advantage of the model is its simplicity in numerical calculations since the most difficult task is to find two distinct real roots for martensite mass fraction $\xi$ in quadratic function (17). It should also be noted that the proposed model can be used for analysis of constrained recovery in SMA wire subjected to compression with only a changing sign of certain constants, i.e., prestain $\varepsilon_{S 0}$, contact strain $\varepsilon_{C}$, stress rate $C$, and taking the plus sign in Eq. (19).

\section{Acknowledgment}

The authors gratefully acknowledge the help of Prof. Hiroyuki Kato from Hokkaido University, who generously provided the experimental data.

\section{References}

[1] M. Kapgan and K.N. Melton, Shape memory alloy tube and pipe couplings. In: Engineering Aspects of Shape Memory Alloys, T.W. Duerig, K.N. Melton, D. Stockel, and C.M. Wayman, Eds., Butterworth-Heinemann, London, pp. 137-148, 1990.

[2] P. Dai and L. Zhou, Investigation on the connecting strength of Fe-Mn-Si-C shape memory alloy pipe coupling, J. Mater. Sci., vol. 41, pp. 3441-3443, 2006.

[3] K.N. Melton, General applications of SMA's and smart materials. In: Shape Memory Materials, K. Otsuka and C.M. Wayman, Eds., Cambridge University Press, Cambridge, chap. 10, 1998.

[4] D. Christ and S. Reese, A finite element model for shape memory alloys considering thermomechanical couplings at large strains, Int. J. Solids Struct., vol. 46, pp. 3694-3709, 2009.

[5] S.M. Russell, Design considerations for nitinol bone staples, J. Mater. Eng. Perform., vol. 18, pp. 831-835, 2009.

[6] K. Shahin, G.P. Zou, and F. Taheri, Shape memory alloy wire reinforced composites for structural damage repairs, Mech. Adv. Mater. Struct., vol. 12, pp. 425-435, 2005.

[7] D.M. Peairs, G. Park, and D.J. Inman, Practical issues of activating self-repairing bolted joints, Smart Mater. Struct., vol. 13, pp. 1414-1423, 2004.

[8] G.R. Edwards, J. Perkins, and J.M. Johnson, Characterizing the shape memory effect potential of Ni-Ti alloys, Scr. Metall., vol. 9, pp. 1167-1171, 1975.

[9] H.A. Mohamed, Determination of the recovery stresses developed by shape memory alloys, J. Mater. Sci., vol. 13, pp. 2728-2730, 1978. 
[10] C. Liang and C.A. Rogers, One-dimensional thermomechanical constitutive relations for shape memory materials, J. Intell. Mater. Sys. Struct., vol. 1, pp. 207-234, 1990.

[11] L.C. Brinson, One-dimensional constitutive behavior of shape memory alloys: Thermomechanical derivation with non-constant material functions and redefined martensite internal variable, J. Intell. Mater. Sys. Struct., vol. 4, pp. 229-242, 1993.

[12] R. Stalmans, L. Delaey, and J. Van Humbeeck, Generation of recovery stresses: Thermodynamic modelling and experimental verification, J. Phys. IV, vol. 7, no. C5, pp. 47-52, 1997.

[13] S. Leclercq and C. Lexcellent, A general macroscopic description of the thermomechanical behavior of shape memory alloys, J. Mech. Phy. Solids., vol. 44, pp. 953-980, 1996.

[14] P. Sittner, D. Vokoun, G.N. Dayananda, and R. Stalmans, Recovery stress generation in shape memory $\mathrm{Ti}_{50} \mathrm{Ni}_{45} \mathrm{Cu}_{5}$ thin wires, Mater. Sci. Eng. A, vol. 286, pp. 298-311, 2000.

[15] H. Kato, N. Inagaki, and K. Sasaki, A one-dimensional modelling of constrained shape memory effect, Acta Mater., vol. 52, pp. 3375-3382, 2004.
[16] G.H. Pan and W.M. Huang, A note on constrained shape memory alloys upon thermal cycling, J. Mater. Sci., vol. 41, pp. 7964-7968, 2006.

[17] F. Kosel and T. Videnic, Generalized plasticity and uniaxial constrained recovery in shape memory alloys, Mech. Adv. Mater. Struct., vol. 14, pp. 3-12, 2007.

[18] K. Nagaya and Y. Hirata, Analysis of a coupling made of shape memory alloy and its dynamic response due to impacts, J. Vibr. Acoust., vol. 114, pp. 297-304, 1992.

[19] T. Videnic, F. Kosel, V. Sajn, and M. Brojan, Biaxial constrained recovery in shape memory alloy rings, J. Intell. Mater. Sys. Struct., vol. 19, pp. 861-874, 2008.

[20] J. Lubliner and F. Auricchio, Generalized plasticity and shape memory alloys, Int. J. Solids Struct., vol. 33, pp. 991-1003, 1996.

[21] F. Auricchio and J. Lubliner, A uniaxial model for shape-memory alloys, Int. J. Solids Struct., vol. 34, pp. 3601-3618, 1997.

[22] C.L. Lagoudas and Z. Bo, Thermomechanical modeling of polycrystalline SMAs under cyclic loading, Part II: Material characterization and experimental results for a stable transformation cycle, Int. J. Eng. Sci., vol. 37, pp. 1141-1173, 1999. 